\title{
Transduction motif analysis of gastric cancer based on a human signaling network
}

\author{
G. Liu*, D.Z. Li*, C.S. Jiang and W. Wang \\ Department of Gastroenterology, Fuzhou General Hospital of Nanjing Command, Fuzhou, China
}

\begin{abstract}
To investigate signal regulation models of gastric cancer, databases and literature were used to construct the signaling network in humans. Topological characteristics of the network were analyzed by CytoScape. After marking gastric cancer-related genes extracted from the CancerResource, GeneRIF, and COSMIC databases, the FANMOD software was used for the mining of gastric cancer-related motifs in a network with three vertices. The significant motif difference method was adopted to identify significantly different motifs in the normal and cancer states. Finally, we conducted a series of analyses of the significantly different motifs, including gene ontology, function annotation of genes, and model classification. A human signaling network was constructed, with 1643 nodes and 5089 regulating interactions. The network was configured to have the characteristics of other biological networks. There were 57,942 motifs marked with gastric cancer-related genes out of a total of 69,492 motifs, and 264 motifs were selected as significantly different motifs by calculating the significant motif difference (SMD) scores. Genes in significantly different motifs were mainly enriched in functions associated with cancer genesis, such as regulation of cell death, amino acid phosphorylation of proteins, and intracellular signaling cascades. The top five significantly different motifs were mainly cascade and positive feedback types. Almost all genes in the five motifs were cancer related, including EPOR, MAPK14, BCL2L1, KRT18, PTPN6, CASP3, TGFBR2, AR, and CASP7. The development of cancer might be curbed by inhibiting signal transductions upstream and downstream of the selected motifs.
\end{abstract}

Key words: Significantly different motifs; Human signaling network; Gastric cancer

\section{Introduction}

Numerous studies have shown that the abnormal transduction of cellular signaling is closely related to differentiation, apoptosis, and proliferation of cells, and to the occurrence, progression, and prognosis of disease (1). According to studies of intercellular protein-protein interaction networks, the regulation of local signaling in normal tissue is different from that in tumors (2). Network motifs are the specific combinations of functional vertices and the basic building blocks of a network. Motifs can react to external stimuli by regulating gene expression. Mining the cancer susceptibility genes, combined network motifs, and gene expression profiles (3) can improve the identification of target genes on tumor metastasis markedly $(4,5)$.

About $90 \%$ of early gastric cancer patients with adequate treatment can survive for more than 5 years and be considered cured; however, the 5-year survival rate of advanced gastric cancer after treatment is less than $5 \%(6)$. Thus, early diagnosis is the key to improving treatment efficacy and increasing survival rate (7).

In this study, in order to screen for gastric cancerrelated genes and then investigate the signal-regulating models, we constructed a human signaling network after integrating information from many databases and references. After analysis of topological properties, we mapped the verified genes onto the network, and mined the cancer-related motifs using three vertices. Finally, we selected the motifs that were significantly different in normal compared with gastric cancer cells. Genes in the significantly different motifs were the screened genes.

\section{Material and Methods}

\section{Gene expression profiles}

The Gene Expression Omnibus (GEO) database (http://www.ncbi.nlm.nih.gov/geo/) is currently the largest

Correspondence: W. Wang, Department of Gastroenterology, Fuzhou General Hospital of Nanjing Command, Fuzhou, 350025, China. Fax: +86-0591-249-59371. E-mail: wangw68@medmail.com.cn

${ }^{*}$ These authors contributed equally to this study.

Received September 15, 2013. Accepted January 13, 2014. First published online April 4, 2014. 
fully public gene expression resource. It provides flexible mining tools that enable users to easily query, filter, inspect and download data within the context of their specific interests (8). We downloaded the gene expression profile data of GSE2685 (9) from GEO, which was based on the GPL80 platform (HU6800; Affymetrix Human Full Length HugeneFL Array) data. A total of 30 samples were available, including primary human advanced gastric cancer tissues $(n=22)$, and noncancerous gastric tissues $(n=8)$. We downloaded the raw data and the probe annotation files from Affymetrix for further analysis. The probe-level data were converted into expression values, $\log _{2}$ transformed, and standardized using the median method (10).

\section{Extraction of gastric cancer-related genes}

Gastric cancer-related genes were extracted from CancerResource (11), GeneRIF (Gene Reference into Function) (12), and COSMIC (Catalogue of Somatic Mutations in Cancer) (13) databases.

\section{Human signaling network construction}

All cellular activities, including division, differentiation, and apoptosis are closely associated with signal transduction. The BioCarta database is the largest collection of information on human signaling pathways. We downloaded all the human signaling pathways from BioCarta (http://www.biocarta.com/genes/Cellsignaling.asp) (14), removed redundant information, and represented all proteins with their corresponding genes. In addition, 10 cancer-related pathways from Cancer CellMap (15) and pathways published by Le and Kwon (16) were also used to construct the signaling network associated with gastric cancer. Gastric cancer-related genes extracted from different databases were then marked into the signaling network. Finally, the network analyzer tool in CytoScape was used to calculate network topological characteristics such as degree distribution and clustering coefficient.

\section{Motif mining in human signaling network}

Many biological networks consist of specific combinations of subnets with frequencies of occurrence that are significantly higher than random. Topological motifs with high frequencies can be used to explain the principles of bio-network organization (17). The fast network motif detection (FANMOD) software (18) was used for motif mining in the human signaling network, because it can handle networks with colors in nodes and edges, and predict the mining time for the whole network with a high operating efficiency.

\section{Screening for significant differences among motifs}

To investigate the differences of motifs in the normal and cancer states, the significant motif difference (SMD) method (19), based on variations of coexpression, was used to calculate the SMD scores of motifs. For a motif
$\left(M_{A}\right)$ with three edges, E1, E2, and E3, the difference score (S) is defined as:

$$
\begin{aligned}
& \mathrm{S}\left(\mathrm{M}_{\mathrm{A}}\right)=\sum_{\mathrm{k}=1}^{\mathrm{n}} \operatorname{abs}\left(\mathrm{E}_{\mathrm{k}}-\mathrm{E}_{\mathrm{k}}^{\prime}\right), \mathrm{n}=3 \\
& \mathrm{E}_{\mathrm{k}}=|\operatorname{Pearson}(\mathrm{X}, \mathrm{Y})|=\left|\frac{\operatorname{cov}(\mathrm{X}, \mathrm{Y})}{\sqrt{\mathrm{D}(\mathrm{X})} \sqrt{\mathrm{D}(\mathrm{Y})}}\right| \\
& \mathrm{E}_{\mathrm{k}}^{\prime}=\left|\operatorname{Pearson}\left(\mathrm{X}^{\prime}, \mathrm{Y}^{\prime}\right)\right|=\left|\frac{\operatorname{cov}\left(\mathrm{X}^{\prime}, \mathrm{Y}^{\prime}\right)}{\sqrt{\mathrm{D}\left(\mathrm{X}^{\prime}\right)} \sqrt{\mathrm{D}\left(\mathrm{Y}^{\prime}\right)}}\right|
\end{aligned}
$$

where $X, Y$ are the gene expression values in the normal state and $X^{\prime}, Y^{\prime}$ are the gene expression values in the cancer state. $E_{k}$ and $E^{\prime}{ }_{k}$ are the absolute values of Pearson correlated coefficients between the two genes connecting by edge $\mathrm{k}$ under normal and cancer states, respectively.

Motifs with SMD scores higher than threshold are the significantly different motifs, and the threshold is set according to the distributions of SMD scores. $\mathrm{P}=0.05$ was selected as the significance threshold.

\section{Functional annotations of significantly different motifs}

Gene ontology (GO) functional annotations (20) of genes in significantly different motifs were performed using the Database for Annotation, Visualization, and Integration Discovery (DAVID) (21). Functions with a corrected $\mathrm{P}$ value false discovery rate (FDR) of less than 0.05 were selected.

\section{Results}

\section{Gastric cancer-related genes}

By screening the expression profiles and extracting from three databases, 5515 and 778 related genes were obtained, respectively.

\section{Human signaling network construction}

The human signaling network was constructed combining the pathways obtained from databases and references. There were 1634 nodes and 5089 regulating interactions, including 2403 activated, 741 inhibited, and 1915 physical interactions in the network (Figure 1).

The integrated network was hypothesized to have the same characteristics, such as small-world, scale-free, and hierarchy as protein-protein interaction networks, and gene networks (22). The CytoScape NetworkAnalyzer was used to calculate the degree distribution (Figure 2A) and clustering coefficient (Figure 2B) of the network. It turned out than the degree distribution followed a power law, and the network had scale-free and small-world characteristics. The average degree was 6.3 , but was 10.5 for gastric cancer-related genes, almost all of which 


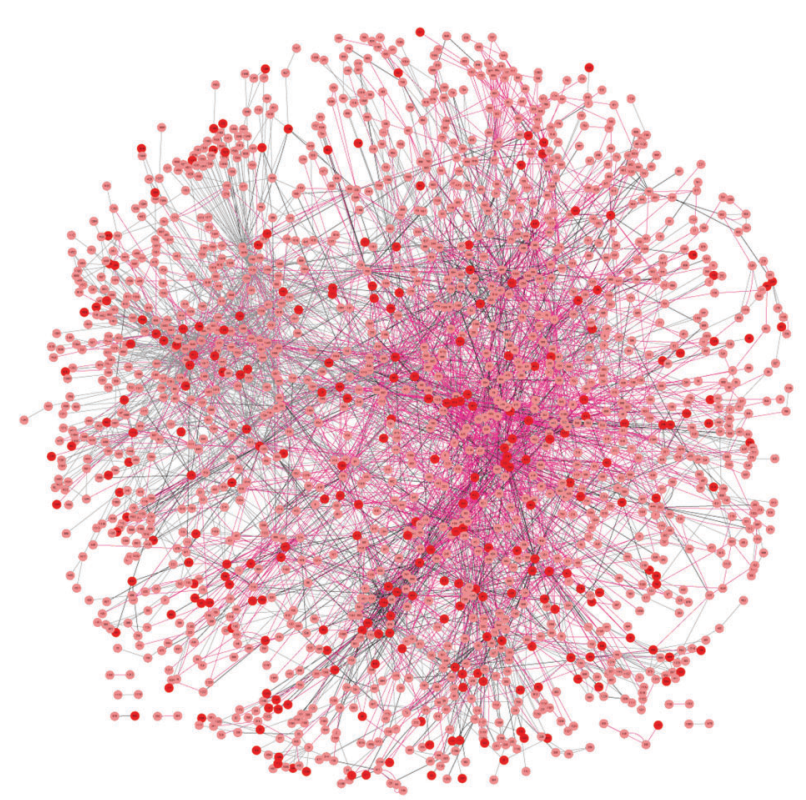

Figure 1. Human signaling network. Light gray lines represent the physical interactions, dark black lines represent the inhibited interactions, and pink lines represent the activated interactions. The dark red nodes are cancer-related genes.

were hub genes in the network (23). As shown in Figure $2 \mathrm{~B}$, the genes with a higher number of neighbors tended to have lower clustering coefficients.

\section{Human signaling network motif mining}

Biological networks are composed of recurring network models, and all models are usually combinations of motifs with three vertices. We conducted the motif mining using the FANMOD software for the gastric cancerrelated motifs with three vertices. The nodes and edges in

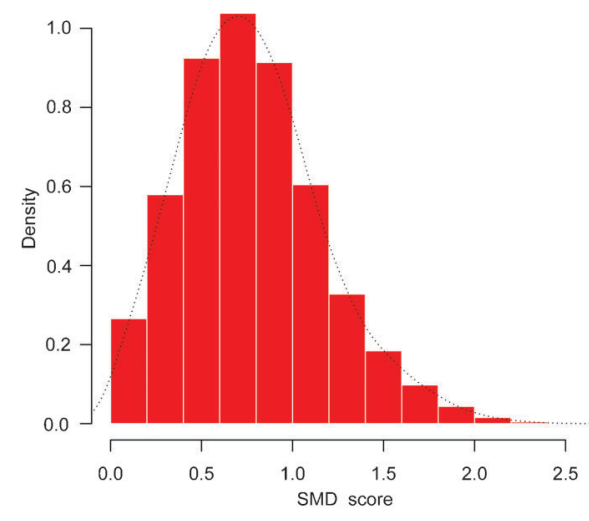

Figure 3. Distribution of significant motif difference (SMD) scores of motifs marked with gastric cancer-related genes.

the network were marked in different colors. In the total of 92 models, 90 were marked with cancer-related genes. Of a total of 69,492 motifs, 57,942 were marked with cancerrelated genes.

\section{Significantly different motif selection}

SMD scores of 57,942 motifs were computed using the gene expression profiles under normal and cancer states. In all, 26,354 motifs were selected with all three genes expressed, and the distributions of these motif scores were normally distributed (Figure 3). The SMD scores in the normal and cancer states were significantly different for 264 motifs $(\mathrm{P}<0.05)$.

\section{Functional annotations of significantly different motifs}

Genes in the significantly different motifs were mainly enriched in functions closely related to the occurrence of cancer, such as regulation of cell death, regulation of
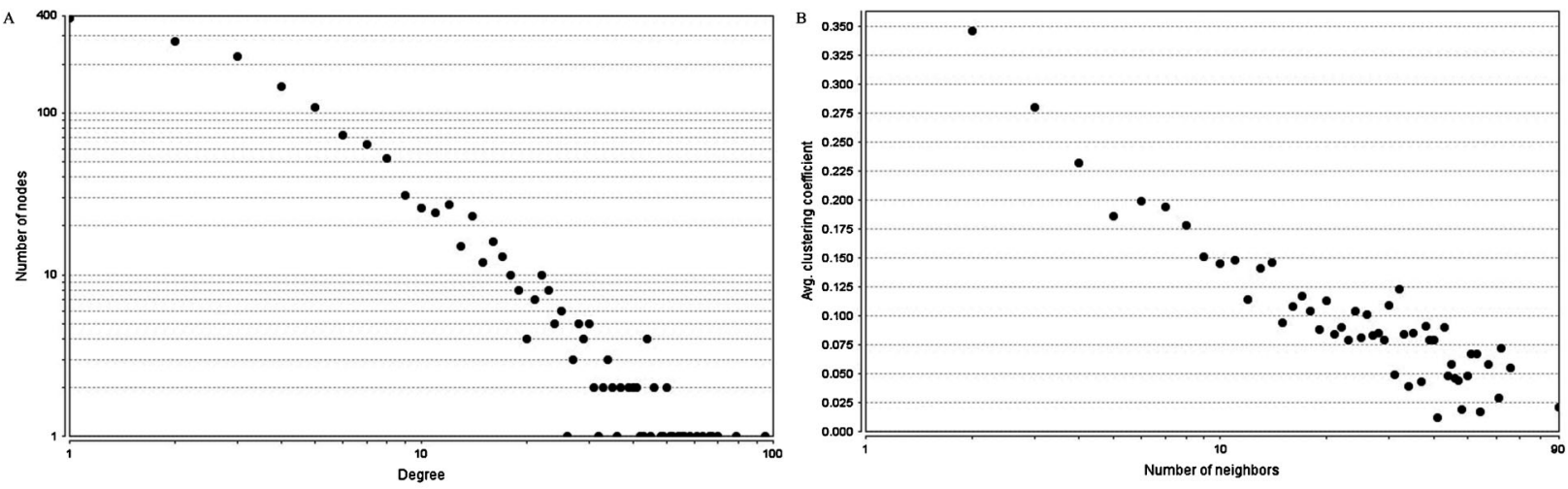

Figure 2. A, Degree distributions of the human signaling network. Numbers of nodes with higher degree were smaller than the other nodes, and all nodes approximated a power-law. $B$, Clustering coefficients distributions of the human signaling network. The average clustering coefficient of all nodes was plotted against the numbers of neighbors, and nodes with smaller coefficients tended to have fewer neighbors. 
programmed cell death, protein amino acid phosphorylation, and intracellular signaling cascades (Table 1). This result confirmed the relationship between the significantly different motifs and gastric cancer.

\section{Type and rank analysis of significantly different motifs}

First, we classified the types of significantly different motifs, and found that the types having more than five motifs were mainly cascades and positive feedback (Figure 4). Next, we ranked the motifs according to their SMD scores (Table 2), and queried for the relationship of genes of the top five motifs with gastric cancer. Among all the genes, only two, NCOR2 (human nuclear corepressor 2) and ARHGEF7 (rho guanine nucleotide exchange factor), were found to have no relation to gastric cancer. The relationships of EPOR (erythropoietin receptor), MAPK14 (mitogen-activated protein kinase 1), BCL2L1 (BCL2-like1), KRT18 (keratin 18), PTPN6 (protein tyrosine phosphatase nonreceptor 6), CASP3 (caspase-3), TGFBR2 (transforming growth factor-beta, TGF $\beta$, type II receptor), $A R$ (adrenergic receptor), and CASP7 (caspase-7) with gastric cancer were already known.

\section{Discussion}

The human signaling network we constructed was very large and could reveal additional signal-associated information about gastric cancer. Analysis of the topological characteristics of the network revealed that gastric cancer-related genes had a higher average degree than that of all the genes taken together, and that most of these cancer-related genes were hub genes in the network. This result further confirmed the importance of cancer-related

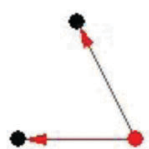

000000111

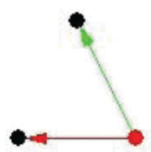

000000211

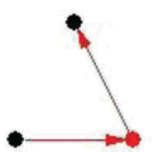

000001101

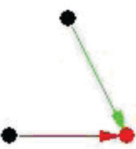

002001001
Figure 4. Models of significantly different motifs. Red arrows are the activated interactions, while green arrows are the inhibited interactions. Black nodes represent normal genes, while red nodes represent gastric cancer-related genes.

genes (23). We also conducted cancer-related motif mining for a better understanding of the mechanisms of cancer occurrence and development. Cascade and positive feedback were the two types of motifs with significantly different normal and cancer state SMD scores, suggesting that they are disrupted in the cancer state, which may promote the speed of signal transduction. Various types of motifs are associated with cell functions. The significance of the cascade type lies in its influence on cell proliferation and differentiation, the negative feedback type participates in an adaptive response, and the positive feedback type can enhance signal robustness $(24,25)$. Thus, efficient signal transduction may be the reason why cancer cells can proliferate so rapidly.

We mapped gene expression values to the signaling network and then screened the significantly different motifs according to differences in coexpression of motif genes between the normal and cancer states. Expression of genes in the selected motifs was mainly enriched in those functions implicated with cancer development, such as regulation of cell death, regulation of programmed cell death, protein amino acid phosphorylation, and intercellular

Table 1. Top 15 functions of genes in the significantly different motifs.

\begin{tabular}{llc}
\hline Category & \multicolumn{1}{c}{ Term } & FDR \\
\hline GOTERM_BP_FAT & GO:0007242-intracellular signaling cascade & $6.64 \mathrm{E}-25$ \\
GOTERM_BP_FAT & GO:0043067-regulation of programmed cell death & $1.27 \mathrm{E}-23$ \\
GOTERM_BP_FAT & GO:0010941-regulation of cell death & $1.55 \mathrm{E}-23$ \\
GOTERM_CC_FAT & GO:0005829-cytosol & $8.74 \mathrm{E}-23$ \\
GOTERM_BP_FAT & GO:0042981-regulation of apoptosis & $3.89 \mathrm{E}-22$ \\
GOTERM_BP_FAT & GO:0010033-response to organic substance & $6.09 \mathrm{E}-22$ \\
GOTERM_BP_FAT & GO:0010604-positive regulation of macromolecule metabolic process & $1.51 \mathrm{E}-21$ \\
GOTERM_BP_FAT & GO:0043065-positive regulation of apoptosis & $5.66 \mathrm{E}-20$ \\
GOTERM_BP_FAT & GO:0043068-positive regulation of programmed cell death & $7.43 \mathrm{E}-20$ \\
GOTERM_BP_FAT & GO:0010942-positive regulation of cell death & $8.89 \mathrm{E}-20$ \\
GOTERM_BP_FAT & GO:0006468-protein amino acid phosphorylation & $3.07 \mathrm{E}-19$ \\
GOTERM_BP_FAT & GO:0007167-enzyme linked receptor protein signaling pathway & $9.14 \mathrm{E}-19$ \\
GOTERM_BP_FAT & GO:0006796-phosphate metabolic process & $5.50 \mathrm{E}-18$ \\
GOTERM_BP_FAT & GO:0006793-phosphorus metabolic process & $5.50 \mathrm{E}-18$ \\
GOTERM_BP_FAT & GO:0031328-positive regulation of cellular biosynthetic process & $6.66 \mathrm{E}-18$ \\
\hline
\end{tabular}

BP: biological process; CC: cellular component; GO: gene ontology; FDR: false discovery rate. 
Table 2. Top 5 motifs ranked by the significant motif difference scores.

\begin{tabular}{lcccc}
\hline Motif & Gene 1 & Gene 2 & Gene 3 & Score \\
\hline 003300001 & EPOR & PTPN6 & KRT18* & 2.503577 \\
000000211 & MAPK14* & NCOR2 & ARHGEF7 & 2.40976 \\
000303001 & PTPN6 & KRT18* & TGFBR2 & 2.390741 \\
000300011 & MAPK14* & NCOR2 & AR & 2.3854 \\
100010231 & BCL2L1* & CASP3 & CASP7 & 2.378815 \\
\hline
\end{tabular}

*Already known genes.

signaling cascades. Recently, studies have shown that amino acids are not only cell signaling molecules but also regulators of gene expression and the protein phosphorylation cascade (26). The signaling pathways of the cellular response to accurate transmission of signals rely on protein phosphorylation and, ultimately, lead to the activation of specific transcription factors that induce the expression of appropriate target genes (27). Extracellular signals are transmitted from the cell membrane to genes in the nucleus via several communication lines known as intracellular signaling pathways, and the transmission of signals through these pathways involves sequential phosphorylation events, in many cases by protein kinases, that are termed kinase cascades (28). Among signal transduction events, protein phosphorylation modulated by protein kinases and phosphatases is an important posttranslational modification event in a variety of cells. Such phosphorylation plays a critical function in signal transduction, cell growth, differentiation, and oncogenesis (29). All the enriched functions in this network were involved in cancer development. Thus, the selected motifs were also related to gastric cancer.

EPOR, MAPK14, BCL2L1, KRT18, PTPN6, CASP3, TGFBR2, $A R$, and CASP7 were genes in the five motifs with the highest SMD scores, and some of them are already known to be gastric cancer related. NCOR2 and $A R H G E F$ were the only two genes for which there have been no reports of a correlation with gastric cancer. EPOR is a member of the cytokine receptor superfamily, and the increased expression of EPOR is a potential, significant prognostic marker in the carcinogenesis, angiogenesis, and progression of gastric cancer (30). The protein tyrosine phosphatase (PTP) family plays an

\section{References}

1. Klinke DJ. Signal transduction networks in cancer: quantitative parameters influence network topology. Cancer Res 2010; 70: 1773-1782, doi: 10.1158/0008-5472.CAN-093234.

2. Jiang Y, Huang T, Chen L, Gao YF, Cai Y, Chou KC. Signal propagation in protein interaction network during colorectal cancer progression. Biomed Res Int 2013; 2013: 287019.

3. Zhang Y, Xuan J, de Los Reyes BG, Clarke R, Ressom HW. important part in the inhibition or control of growth, and members may exert oncogenic functions (31). Several studies have detected aberrant DNA methylation of the PTPN6 gene in gastric cancer $(32,33)$. TGFBR2, a constitutively active kinase, is reported to play a tumor suppressor role in the TGF $\beta$ pathway in gastric cancer (34). Studies have also detected the relevance of $A R(35)$, CASP3 (36), and CASP7 (37) with gastric cancer. NCOR2, which participates in a corepressor complex resulting in chromatin condensation, is involved with many cancers (38). It promotes the deacetylation of histone to silence genes. In addition, ARHGEF7, also known as PAK-interacting exchange factor, participates in the activation of Ras family genes (39). Based on these identifications, even though there is no direct evidence, NCOR2 and ARHGEF may be the latent gastric cancerrelated genes.

Gastric cancer is a common, fatal malignancy worldwide. At present, therapeutic decisions are based on clinical and pathological parameters, including age, tumor-involved lymph nodes, metastases, stage, and histological grade. Although useful, these factors often fail to differentiate more aggressive tumor types from less aggressive types (40). As a result, there is an urgent need to find specific markers. If motifs, as functional units, can be used as biomarkers, then the diagnostic efficiency will be greatly increased. We could then find the locations of the already known cancer-related genes in a motif, and see which genes they affect and which genes affect them. The development of cancers might then be suppressed by inhibiting the signal transductions of their upstream and downstream genes with new potential drugs for gastric cancer.
Network motif-based identification of breast cancer susceptibility genes. Conf Proc IEEE Eng Med Biol Soc 2008; 2008: 5696-5699.

4. Kim MS, Kim JR, Kim D, Lander AD, Cho KH. Spatiotemporal network motif reveals the biological traits of developmental gene regulatory networks in Drosophila melanogaster. BMC Syst Biol 2012; 6: 31, doi: 10.1186/ 1752-0509-6-31. 
5. Liang AJ, Hong Y, Sun Y, Gao MZ, Zhao XM. The regulation network and network motif analysis in ovarian cancer. Eur $\mathrm{J}$ Gynaecol Oncol 2013; 34: 170-174.

6. Ali Z, Deng Y, Ma C. Progress of research in gastric cancer. J Nanosci Nanotechnol 2012; 12: 8241-8248, doi: 10.1166/ jnn.2012.6692.

7. Pietrantonio F, de Braud BF, Da Prat V, Perrone F, Pierotti MA, Gariboldi M, et al. A review on biomarkers for prediction of treatment outcome in gastric cancer. Anticancer Res 2013; 33: 1257-1266.

8. Barrett T, Troup DB, Wilhite SE, Ledoux P, Rudnev D, Evangelista $C$, et al. NCBI GEO: mining tens of millions of expression profiles - database and tools update. Nucleic Acids Res 2007; 35: D760-D765, doi: 10.1093/nar/gkl887.

9. Hippo Y, Taniguchi H, Tsutsumi S, Machida N, Chong JM, Fukayama M, et al. Global gene expression analysis of gastric cancer by oligonucleotide microarrays. Cancer Res 2002; 62: 233-240.

10. Garbett K, Ebert PJ, Mitchell A, Lintas C, Manzi B, Mirnics $\mathrm{K}$, et al. Immune transcriptome alterations in the temporal cortex of subjects with autism. Neurobiol Dis 2008; 30: 303311, doi: 10.1016/j.nbd.2008.01.012.

11. Ahmed J, Meinel T, Dunkel M, Murgueitio MS, Adams R, Blasse C, et al. CancerResource: a comprehensive database of cancer-relevant proteins and compound interactions supported by experimental knowledge. Nucleic Acids Res 2011; 39: D960-D967, doi: 10.1093/nar/gkq910.

12. Jimeno-Yepes AJ, Sticco JC, Mork JG, Aronson AR. GeneRIF indexing: sentence selection based on machine learning. $B M C$ Bioinformatics 2013; 14: 171, doi: 10.1186/1471-2105-14-171.

13. Forbes SA, Tang G, Bindal N, Bamford S, Dawson E, Cole $C$, et al. COSMIC (the Catalogue of Somatic Mutations in Cancer): a resource to investigate acquired mutations in human cancer. Nucleic Acids Res 2010; 38: D652-D657, doi: 10.1093/nar/gkp995.

14. Hu P, Xu W, Cheng L, Xing X, Paterson AD. Pathway-based joint effects analysis of rare genetic variants using Genetic Analysis Workshop 17 exon sequence data. BMC Proc 2011; 5 (Suppl 9): S45, doi: 10.1186/1753-6561-5-S9-S45.

15. Croft D, O'Kelly G, Wu G, Haw R, Gillespie M, Matthews L, et al. Reactome: a database of reactions, pathways and biological processes. Nucleic Acids Res 2011; 39: D691D697, doi: 10.1093/nar/gkq1018.

16. Le DH, Kwon YK. The effects of feedback loops on disease comorbidity in human signaling networks. Bioinformatics 2011; 27: 1113-1120, doi: 10.1093/bioinformatics/btr082.

17. Milo R, Shen-Orr S, Itzkovitz S, Kashtan N, Chklovskii D, Alon U. Network motifs: simple building blocks of complex networks. Science 2002; 298: 824-827, doi: 10.1126/ science.298.5594.824

18. Wernicke S, Rasche F. FANMOD: a tool for fast network motif detection. Bioinformatics 2006; 22: 1152-1153, doi: 10.1093/bioinformatics/btl038.

19. Ficklin SP, Feltus FA. Gene coexpression network alignment and conservation of gene modules between two grass species: maize and rice. Plant Physiol 2011; 156: 12441256, doi: 10.1104/pp.111.173047.

20. Harris MA, Clark J, Ireland A, Lomax J, Ashburner M, Foulger R, et al. The Gene Ontology (GO) database and informatics resource. Nucleic Acids Res 2004; 32: D258D261, doi: 10.1093/nar/gkh066.
21. Dennis G Jr, Sherman BT, Hosack DA, Yang J, Gao W Lane HC, et al. DAVID: Database for annotation, visualization, and integrated discovery. Genome Biol 2003; 4: 3, doi: 10.1186/gb-2003-4-5-p3.

22. Hu G, Zhou J, Yan W, Chen J, Shen B. The topology and dynamics of protein complexes: insights from intra-molecular network theory. Curr Protein Pept Sci 2013; 14: 121-132, doi: 10.2174/1389203711314020004.

23. Zhang J, Yang Y, Wang Y, Zhang J, Wang Z, Yin M, et al. Identification of hub genes related to the recovery phase of irradiation injury by microarray and integrated gene network analysis. PLoS One 2011; 6: e24680, doi: 10.1371/journal. pone.0024680.

24. Mangan S, Alon U. Structure and function of the feedforward loop network motif. Proc Natl Acad Sci U S A 2003; 100: 11980-11985, doi: 10.1073/pnas.2133841100.

25. Akiva E, Friedlander G, Itzhaki Z, Margalit H. A dynamic view of domain-motif interactions. PLoS Comput Biol 2012; 8: e1002341, doi: 10.1371/journal.pcbi.1002341.

26. Wu G. Amino acids: metabolism, functions, and nutrition Amino Acids 2009; 37: 1-17, doi: 10.1007/s00726-0090269-0.

27. Viatour P, Merville MP, Bours V, Chariot A. Phosphorylation of NF-kappaB and IkappaB proteins: implications in cancer and inflammation. Trends Biochem Sci 2005; 30: 43-52, doi: 10.1016/j.tibs.2004.11.009.

28. Yoon S, Seger R. The extracellular signal-regulated kinase: multiple substrates regulate diverse cellular functions. Growth Factors 2006; 24: 21-44.

29. Wu CW, Chi CW, Lin WC. Gastric cancer: prognostic and diagnostic advances. Expert Rev Mol Med 2002; 4: 1-12, doi: $10.1017 / S 1462399402004337$.

30. Wang L, Li HG, Xia ZS, Wen JM, LV J. Prognostic significance of erythropoietin and erythropoietin receptor in gastric adenocarcinoma. World J Gastroenterol 2011; 17: 3933-3940, doi: 10.3748/wjg.v17.i34.3933.

31. Julien SG, Dube N, Hardy S, Tremblay ML. Inside the human cancer tyrosine phosphatome. Nat Rev Cancer 2011; 11: 35-49, doi: 10.1038/nrc2980.

32. Stebbing J, Lit LC, Zhang $\mathrm{H}$, Darrington RS, Melaiu $\mathrm{O}$, Rudraraju B, et al. The regulatory roles of phosphatases in cancer. Oncogene 2014; 33: 939-953, doi: 10.1038/ onc. 2013.80

33. Shin CM, Kim N, Park JH, Kang GH, Kim JS, Jung HC, et al. Prediction of the risk for gastric cancer using candidate methylation markers in the non-neoplastic gastric mucosae. J Pathol 2012; 226: 654-665, doi: 10.1002/path.2990.

34. Jin G, Wang L, Chen W, Hu Z, Zhou Y, Tan Y, et al. Variant alleles of TGFB1 and TGFBR2 are associated with a decreased risk of gastric cancer in a Chinese population. Int J Cancer 2007; 120: 1330-1335, doi: 10.1002/ijc.22443.

35. Shi M, Yang Z, Hu M, Liu D, Hu Y, Qian L, et al. Catecholamine-induced beta2-adrenergic receptor activation mediates desensitization of gastric cancer cells to trastuzumab by upregulating MUC4 expression. $\mathrm{J}$ Immunol 2013; 190: 5600-5608, doi: 10.4049/jimmunol.1202364

36. Xu MY, Lee DH, Joo EJ, Son KH, Kim YS. Akebia saponin PA induces autophagic and apoptotic cell death in AGS human gastric cancer cells. Food Chem Toxicol 2013; 59: 703-708, doi: 10.1016/j.fct.2013.06.059.

37. Li Q, Zhang H, Tan C, Peng W, Ren G, Jia B, et al. AdHu5- 
apoptin induces G2/M arrest and apoptosis in p53-mutated human gastric cancer SGC-7901 cells. Tumour Biol 2013; 34: 3569-3577, doi: 10.1007/s13277-013-0936-3.

38. van Agthoven T, Sieuwerts AM, Veldscholte J, Meijer-van Gelder ME, Smid M, Brinkman A, et al. CITED2 and NCOR2 in anti-oestrogen resistance and progression of breast cancer. Br J Cancer 2009; 101: 1824-1832, doi: 10.1038/sj. bjc. 6605423.

39. Hua KT, Tan CT, Johansson G, Lee JM, Yang PW, Lu HY, et al. $\mathrm{N}$-alpha-acetyltransferase 10 protein suppresses cancer cell metastasis by binding PIX proteins and inhibiting Cdc42/Rac1 activity. Cancer Cell 2011; 19: 218-231, doi: 10.1016/j.ccr.2010.11.010.

40. Laimer K, Fong D, Gastl G, Obrist P, Kloss F, Tuli T, et al. EpCAM expression in squamous cell carcinoma of the oral cavity: frequency and relationship to clinicopathologic features. Oral Oncol 2008; 44: 72-77, doi: 10.1016/j.oraloncology. 2007.01.008 